\title{
Les gynécomasties à Dakar (à propos de 30 cas)
}

\author{
E.H. SIDIBE \\ Clinique Médicale II - Service de Médecine Interne Centre Marc SANKALE - FANN - DAKAR (Sénégal) \\ Université de Dkar et Université Paris 7
}

\section{RÉSUMÉ}

Les gynécomasties sont peu étudiées à Dakar. Notre objectif est d'étudier les aspects sociodémographiques, cliniques et étiologiques des gynécomasties à Dakar. Chez des patients de notre Service, une étude rétrospective (période 1980-1996) a porté sur l'état civil (âge, ethnie, origine géographique), les aspects cliniques (topographie, ancienneté, douleur, galactorrhée, caractères sexuels secondaires, signes associés) et les aspects étiologiques. Nous avons 30 dossiers de gynécomastie, soit $0,93 \%$ des dossiers des consultants en Médecine. La moyenne d'âge est de 22 ans ; $36 \%$ des patients consultent à l'adolescence, la moitié entre 21 et 30 ans, et $6 \%$ après l'âge de 30 ans. Une prédominance des ouoloffs (11/30) par rapport aux autres ethnies existe. Nos patients sont d'origine essentiellement suburbaines (26/30). Chez $30 \%$ des patients la gynécomastie est récente (maximum 1 an). Dans $43 \%$, elle existe depuis 2 à 5 ans et dans $20 \%$ depuis 6 à 10 ans. Seuls $6 \%$ des patients consultent très tard. Dans la moitié des cas, il y a bilatéralité avec prédominance gauche $(6 / 16)$. Les cas unilatéraux sont gauches dans $26 \%$ des cas et droits dans $20 \%$. La douleur existe dans $45,85 \%$ des cas, la galactorrhée, 5 fois. L'atrophie testiculaire dans deux cas est sans impact sur les caractères sexuels secondaires et la fonction sexuelle. L'obésité chez 4 patients, est gynoïde dans 3 cas, mixte une fois. Dans plus de la moitié des cas, la période pubertaire est concernée et dans $\mathbf{7 6 , 6 6} \%$ des cas, l'adolescence. L'échographie testiculaire chez 7 patients est normale ; de même que la radiographie pulmonaire et la selle turcique. Notre étude montre une prédilection de la gynécomastie pour l'adolescence $(\mathbf{7 6 , 6} \%$ des cas) et une prédominance de la population suburbaine faisant évoquer le rôle d'une adaptation environnementale.

Mots-Clés : Gynécomastie, Santé de l'adolescent, Afrique, Sénégal.

\section{INTRODUCTION}

La gynécomastie résume la plus grande partie de la pathologie mammaire de l'homme puisque les tumeurs bénignes ou malignes du sein sont exceptionnelles. La difficulté est de savoir où finit la physiologie et où commence la pathologie. Le développement mammaire est habituel dans les deux sexes à la naissance. $60 \%$ des adolescents présentent une gynécomastie au moment de la puberté avec une incidence maximale qui se situe vers l'âge de 14 ans. Cette dernière va régresser habituelle-

Correspondance : El-Hassane Sidibé, Clinique Médicale II - Service de Médecine Interne, Centre Marc SANKALE - B.P. 5062 - FANN - DAKAR (Sénégal) 
ment en l'espace de 1 à 3 ans. On a pu observer une gynécomastie chez $33 \%$ de sujets de 20 à 25 ans [3, 12]. À partir de la trentaine, la gynécomastie est retrouvée de plus en plus souvent au fil des ans. Dans une étude portant sur 447 autopsies de sujets âgés de 59 ans, en moyenne, la gynécomastie existait dans $40 \%$ des cas [15]. Après 70 ans, ce pourcentage peut dépasser $70 \%$ [11]. Cette fréquence, pour le moins surprenante, évoque la possibilité d'une prédominance raciale, puisque ces données ont été observées dans la population d'origine caucasienne $[1,3]$. Dans la littérature, les formes idiopathiques des gynécomasties sont largement prédominantes chez l'adolescent et même chez l'adulte jeune; cependant, GAUTIER et collaborateurs retrouvent $10 \%$ de formes secondaires chez 80 adolescents [6] porteurs de gynécomasties apparemment idiopathiques, d'où la nécessité d'une bonne approche diagnostique.

Lorsqu'elle survient en période pubertaire, isolée, il s'agit probablement d'une gynécomastie idiopathique : toutefois il est nécessaire de proposer une stratégie diagnostique de prise en charge pour ne pas passer à côté de formes secondaires. L'objectif de notre travail est d'étudier les aspects socio-démographiques pouvant être spécifiques à notre site d'étude dakarois (Sénégal).

\section{MATERIEL ET METHODES}

Cette étude a été effectuée chez des patients du Service de Médecine Interne de l'Hôpital Abass NDAO de Dakar.

Il s'agit d'une étude rétrospective de tous les dossiers de gynécomastie durant la période 1980-1996. Pour chaque dossier, ont été étudiés :

Létat civil (âge, ethnie, origine géographique).

Les aspects cliniques (topographie, ancienneté, douleur, galactorrhée, caractères sexuels secondaires, signes associés).

Les aspects étiologiques.

\section{RESULTATS}

\section{A - Résultats globaux}

Sur 3217 dossiers de consultants en médecine, nous avons trouvé 30 dossiers de gynécomastie, soit $0,93 \%$ sur une période de 16 ans.

\section{B - Etude analytique \\ 1 - Etat civil \\ $\boldsymbol{a}$ - L'âge (Tableau 1)}

Lâge moyen des patients au moment de la consultation est de 22 ans, les extrêmes variant de 14 à 38 ans. Seul $36 \%$ des patients consultent à l'adolescence, la moitié entre 21 et 30 ans, et $6 \%$ après l'âge de 30 ans.

\section{b - L'ethnie (Tableau 2)}

Chez 4 patients, l'ethnie n'a pas été précisée, il existe une prédominance des ouolofs $11 / 30$ par rapport aux autres ethnies.

\section{c - L'origine géographique (Tableau 3)}

Nos patients sont d'origine essentiellement suburbaine : $26 / 30$

\section{2 - Les aspects cliniques}

\section{a - L'ancienneté (Tableau 4)}

Chez $30 \%$ des patients, la gynécomastie est récente, c'est-à-dire évoluant depuis au maximum 1 an. Dans $43 \%$, elle évolue depuis 2 à 5 ans, et dans $20 \%$ entre 6 ans et 10 ans. Seul $6 \%$ des patients consultent très tard quand la gynécomastie évolue déjà depuis plus de 11 ans.

\section{b - La topographie (Tableau 5)}

Dans plus de la moitié des cas, la gynécomastie est bilatérale mais avec une prédominance gauche (6/16). Elle est unilatérale gauche dans $26 \%$ des cas et unilatérale droite dans $20 \%$ des cas.

\section{c - Les signes associés}

La douleur : recherchée chez 24 patients, elle a été retrouvée dans $45,8 \%$ des cas à un moment quelconque de l'évolution.

La galactorrhée : recherchée chez 27 patients, elle a été retrouvée 5 fois à l'interrogatoire, cependant elle n'a été objectivée à l'examen qu'une seule fois.

Latrophie testiculaire : deux cas d'atrophie testiculaire ont été signalés chez des patients âgés de 16 et 24 ans, cependant sans impact sur les caractères sexuels secondaires et la fonction sexuelle. 
Tableau 1. Répartition selon l'âge

\begin{tabular}{lccc}
\hline Tranche d'âge & $\mathbf{1 0 - 2 0}$ ans & $\mathbf{2 1 - 3 0}$ ans & $>\mathbf{3 0}$ ans \\
Nombre & 11 & 17 & 2 \\
Pourcentage & $36,6 \%$ & $56,66 \%$ & $6,66 \%$ \\
\hline
\end{tabular}

Tableau 2. Répartition en fonction de l'ethnie

\begin{tabular}{lccccc}
\hline Ethnie & Ouolofs & Sérères & Diolas & Mandingues & Pulhars \\
Nombre de patients & 11 & 2 & 3 & 7 & 3 \\
\hline
\end{tabular}

Tableau 3. Répartition en fonction de l'origine géographique

\begin{tabular}{lccc}
\hline Origine & Rurale & Suburbaine & Urbaine \\
Nombre & 4 & 26 & 0 \\
\hline
\end{tabular}

Tableau 4. Ancienneté de la gynécomastie

\begin{tabular}{lcccc}
\hline Ancienneté & $\leq \mathbf{1}$ an & $\mathbf{2 - 5}$ ans & $\mathbf{6 - 1 0}$ ans & $\geq \mathbf{1 1}$ ans \\
Nombre & 9 & 13 & 6 & 2 \\
Pourcentage & $30 \%$ & $43,33 \%$ & $20 \%$ & $6,6 \%$ \\
\hline
\end{tabular}

Tableau 5 : Répartition selon la topographie des gynécomasties

\begin{tabular}{lccc}
\hline Siège & Gauche & Droite & Bilatérale \\
Nombre & 8 & 6 & 16 \\
Pourcentage & $26,26 \%$ & $20 \%$ & $53,33 \%$ \\
\hline
\end{tabular}


L'obésité : signalée chez 4 patients, elle est gynoïde dans 3 cas, mixte une fois.

Dans la plupart des cas, la gynécomastie apparaît donc cliniquement isolée.

\section{3 - Les aspects étiologiques}

\section{a - L'âge d'apparition de la gynécomastie} (Tableau 6)

Chez plus de la moitié des patients, la gynécomastie s'est installée durant la période pubertaire (10 - 17 ans) et dans $76,6 \%$ des cas durant l'adolescence (0 - 21 ans).

Il y a une prédominance des gynécomasties apparaissant à la période pubertaire surtout lorsque la gynécomastie est cliniquement isolée.

\section{$b$ - Les antécédents}

Aucun cas de pathologie testiculaire n'a été retrouvé dans les antécédents, ni de traumatisme testiculaire même chez les 2 patients chez qui une atrophie des testicules a été signalée à l'examen. Une notion de prise médicamenteuse est signalée chez 3 patients, il s'agit de la prise d'anti-tuberculeux, d'un traitement anti-hansénien, et de la prise de psychotrope qui a été cependant instituée après la survenue de la gynécomastie.

Nous ne retrouvons aucun cas de gynécomastie familiale.

\section{c - L'exploration paraclinique}

Demandée systématiquement, elle n'a été effective que chez très peu de patients du fait du coût élevé notamment de l'exploration hormonale : ainsi la prolactinémie n'a été dosée que chez 4 patients. Les taux sont revenus normaux 2 fois, supérieurs à la normale 1 fois mais ne dépassant pas un taux d'hyperprolactinémie fonctionnelle (inférieurs à $200 \mathrm{ng} / \mathrm{ml}$ ), et inférieurs à la normale une fois.

Le dosage des gonadostimulines FSH-LH réalisé chez 3 patients est revenu normal dans tous les cas. Il en est de même au dosage des œstrogènes.

L'exploration radiologique comporte une échographie testiculaire qui a été demandée chez 7 patients et est revenue normale dans tous les cas ; une radiographie pulmonaire, ainsi qu'une radiographie de la selle turcique demandées respectivement chez 6 et 7 patients sont revenues sans anomalies.

\section{DISCUSSION}

Notre étude n'est qu'un préliminaire à l'analyse des gynécomasties à Dakar en particulier et en Afrique noire en général. Notre travail a une portée limitée du fait de la taille de la série étudiée et du caractère rétrospectif de l'étude. Toutefois, notre analyse des gynécomasties à Dakar permet une première approche.

L'âge jeune des consultants (seuls 2 patients ou plus de 30 ans) est en partie le reflet d'une caractéristique générale non pas tant de la population de recrutement de l'hôpital qui est plutôt de type adulte mais à l'image de la population dakaroise mais aussi du Sénégal dont les différents recensements témoignent d'une population très jeune (âgée de moins de 20 ans dans une proportion de plus de $50 \%$ ). De même, la répartition ethnique reproduit celle de la population dakaroise qui ne diffère pas de la population générale sénégalaise (ouolofs : $35 \%$; sérères : $25 \%$, pulars : $30 \%$; diolas : $5 \%$; madingues : $5 \%$ ) selon les différents recensements. En revanche, la prédominance des suburbains est un facteur particulier qui caractérise la socio-démographie des gynécomasties dans notre cadre d'étude sénégalais. Ceci mérite davantage de réflexion car soulevant le rôle possible de l'environnement (nutrition, culture... ) entre autres hypothèses et les facteurs multiples d'adaptation (stress) de cette population essentiellement d'extraction rurale et nouvellement migratrice dans une nouvelle ville en pleine expansion.

Tableau 6 : Répartition selon l'âge du patient lors de l'apparition de la gynécomastie

\begin{tabular}{lccc}
\hline Age & $\mathbf{1 0 - 1 7}$ ans & $\mathbf{1 8 - 2 1}$ ans & $>\mathbf{2 1}$ ans \\
Nombre & 16 & 7 & 7 \\
Pourcentage & $53,34 \%$ & $23,33 \%$ & $23,33 \%$
\end{tabular}


Quant à l'ancienneté des gynécomasties, elle dépasse 2 ans le plus souvent car seul $1 / 3$ des patients consultent avant ce délai qui habituellement permettrait la régression spontanée de certaines formes dites physiologiques $[3,11]$.

Dans certaines séries de la littérature, la fréquence de l'affection est estimée à $40 \%$ entre 12 et 16 ans voire $65 \%$ au cours de la 14 ème année [14] et $30 \%$ chez l'adulte jeune au cours des $2^{\text {ème }}$ et $3^{\text {ème }}$ décennies [5]. Il s'agit donc essentiellement d'une affection de l'adolescent et de l'adulte jeune.

À cet âge; les gynécomasties sont prépondérantes, $90 \%$ des patients de notre série sont porteurs d'une gynécomastie apparemment physiologique, ce qui est conforme aux données de la littérature [11] ; cependant ceci ne devrait pas faire méconnaitre les formes secondaires. En effet, GAUTIER et coll. ont retrouvé dans leur série $10 \%$ de formes secondaire et dans certaines séries les formes iatrogéniques représentent plus de $60 \%$ des cas [5]. Ce qui n'a pas été le cas dans notre série où la notion de prise d'un médicament pouvant entraîneur une gynécomastie n'a été retrouvée que chez 3 patientes $(10 \%)$. GAUTIER et coll. ont retrouvé une cause iatrogène 7 fois chez 80 patients [6].

La recherche d'une cause tumorale notamment testiculaire, doit demeurer la hantise du médecin, même chez l'adolescent [4], et surtout chez l'adulte jeune [8] grâce à l'évaluation clinique minutieuse et échographique. En effet, une gynécomastie peut être révélatrice d'une tumeur restée longtemps ignorée : 1 patient dans la série de GAUTIER et coll. [6] présentait des anomalies compatibles avec une tumeur testiculaire. D'où la nécessité d'une enquête rigoureuse clinique et d'une exploration paraclinique bien stratifiée surtout dans notre pays où le coût élevé des examens hormonaux constitue un frein à l'enquête étiologique.

Diverses méthodes thérapeutiques sont utilisées dans les gynécomasties physiologiques pour le confort du malade essentiellement $[2$, $7,9,10,13]$.

\section{CONCLUSION}

La gynécomastie est une pathologie surtout fréquente chez l'adolescent et l'adulte jeune, cependant la recherche d'une cause secondaire surtout tumorale testiculaire doit demeurer la hantise du médecin.

Dans plus de la moitié de nos cas, la période pubertaire est concernée et dans $76,7 \%$ des cas l'adolescence.

L'échographie testiculaire chez 7 patients est normale, de même que la radiographie pulmonaire et la selle turcique. La prédominance de la population suburbaine particularise nos cas et nous fait évoquer entre autres hypothèses le rôle d'une adaptation à l'environnement (nutrition, culture, stress... ).

Remerciements : L'auteur souhaite remercier A. Sarr, A. Ndoye-Diop

\section{RÉFÉRENCES}

1. BANNAYAN G.A., HAJDU S.I. : Gynecomastia: clinicopathologic study of 351 cases. Am. J. Clin. Pathol., 1972, $57: 431-437$.

2. BELKENGREN R., SAPALA S. : Pediatric management problems. Physiologic gynecomastia. Pediatr. Nurs., 1995, $21:$ 476-477.

3. BERCOVICI J.P., MAUDELONDE Th. : Les gynécomasties. Encycl. Méd. Chir., Paris, Glandes-Nutrition, 10034, $\mathrm{G}^{10} 2-1983$.

4. BERENSZTEIN E., BELGOROSKY A., DE DAVILA M.T., RIVAROLA M.A. : Testicular steroid biosynthesis in a by with a larger cell calcifying Sertoli cell tumor producing prepubertal gynecomastia. Steroids, $1995,60: 220-225$.

5. CARLSON H.E. : Current concepts. Gynecomastia. N. Engl. J. Med., 1981, 303 : 795-799.

6. GAUTIER D, LASFRAQUE J.J., LEJEUNE J., DRIEU M., FROMANTIN M. : Gynécomasties de l'adulte jeune. Stratégie diagnostique : réflexion à propos de 80 observations. Rev. Franç. Endocrinol. Clin., 1982, $23:$ 235-241.

7. GLASS A.R. : Gynecomastia. Endocrinol. Metab. Clin. North Am., 1994, 23 : 825-837.

8. LEMACK GE, POPPAS D.P., VAUGHAN D. Jr. : Urologic causes of gynecomastia: approach to diagnosis and management. Urology, 1995, $45: 313-319$.

9. MORSELI P.G. : "Pull through" : a new technique for breast reduction in gynecomastia. Plast. Reconstr. Surg., 1996, $97:$ 450-454.

10. MURPHY T.P., EHRLICHMAN R.J., SECKEL B.R. : 
Nipple placement in simple mastectomy with free nipple grafting for severe gynecomastia. Plast. Reconstr. Surg., 1994; 94: 818-823.

11. NYDICK M., BUSTOS J., DALE J.H., RAWSON KW. : Gynecomastia in adolescent boys. J.A.M.A., 1961, $178: 449-454$.

12. RIFKA S.M., PITA J.C., VIGERSKY R.A., WILSON Y.A., LORIAUX D.L. : Interaction of digitalis and spironolactone with human sex steroid receptor. J. Clin. Endocrinol. Metab., 1977, 46 : 338-344.

13. ROSENBERG G.J. : A new cannula, for suction removal of parenchymal tissue of gynecomastia. Plast. Reconstr. Surg., 1994, 94 : 548-551.

14. ROUX S. : Conduite à tenir en cas de gynécomastie. Rev. Prat., 1986, 346 : 25-28.

15. WILSON J.D., AIMAN J., MAC DONALD P.C. : The pathogenesis of gynecomastia. Adv. Intern. Med., $1980,25: 1-32$.

\begin{abstract}
Gynecomastia in Dakar: a series of 30 cases
\end{abstract}

E.H. SIDIBE

Gynecomastia has been rarely studied in Dakar. The objective of this study is to analyze the socio-demographic, clinical and etiologic aspects of gynecomastia in Dakar. A retrospective survey of our patients, from 1980 to 1996, reported age, ethnic and geographic origin, clinical aspects (site, duration, pain, galactorrhea, secondary sexual characteristics, associated signs) and etiologic aspects. Thirty cases of gynecomastia were observed, representing $0.93 \%$ of all patients attending the Department of Internal Medicine. These patients had a mean age of 22 years; $36 \%$ of patients consulted during adolescence, and one half patients consulted between the ages of 21 and 30 years; the majority of patients were Ouolofs. Most patients were suburban (26/30). Gynecomastia was recent (less than 1 year) in $30 \%$ of cases, had been present for 2 to 5 years in $43 \%$ of cases and had been present for 6 to 10 years in $20 \%$ of cases. One half of patients had bilateral gyne- comastia with left-sided predominance $(6 / 16)$. Unilateral cases were left-sided in $26 \%$ of cases and right-sided in $20 \%$ of cases. $45.8 \%$ of cases experienced pain. Galactorrhea was observed in 5 cases. The testicular atrophy observed in two cases had no impact on secondary sexual characteristics and sexual function. Obesity was observed in 4 patients: gynoid in 3 cases and mixed in 1 case. More than one half of all cases presented during puberty and $76.66 \%$ presented during adolescence. Testicular ultrasonography, performed in 7 patients, was normal; chest $x$-ray and sella turcica were also normal. Our study shows that gynecomastia of adolescence represents $76 \%$ of all cases and the high frequency of suburban population indicated a possible role of environmental adaptation.

Key-words: Gynecomastia, Adolescent health, Senegal, Africa. 